This item was submitted to Loughborough's Research Repository by the author.

Items in Figshare are protected by copyright, with all rights reserved, unless otherwise indicated.

\title{
Normal incidence X-ray standing wave study of Fe on $\mathrm{Cu}(111)$
}

PLEASE CITE THE PUBLISHED VERSION

http://dx.doi.org/10.1016/S0039-6028(02)02343-9

PUBLISHER

(c) Elsevier

VERSION

AM (Accepted Manuscript)

LICENCE

CC BY-NC-ND 4.0

REPOSITORY RECORD

Butterfield, Martin T., and Michael D. Cropper. 2019. "Normal Incidence X-ray Standing Wave Study of Fe on $\mathrm{Cu}(111)$ ". figshare. https://hdl.handle.net/2134/10740. 
This item was submitted to Loughborough's Institutional Repository (https://dspace.lboro.ac.uk/) by the author and is made available under the following Creative Commons Licence conditions.

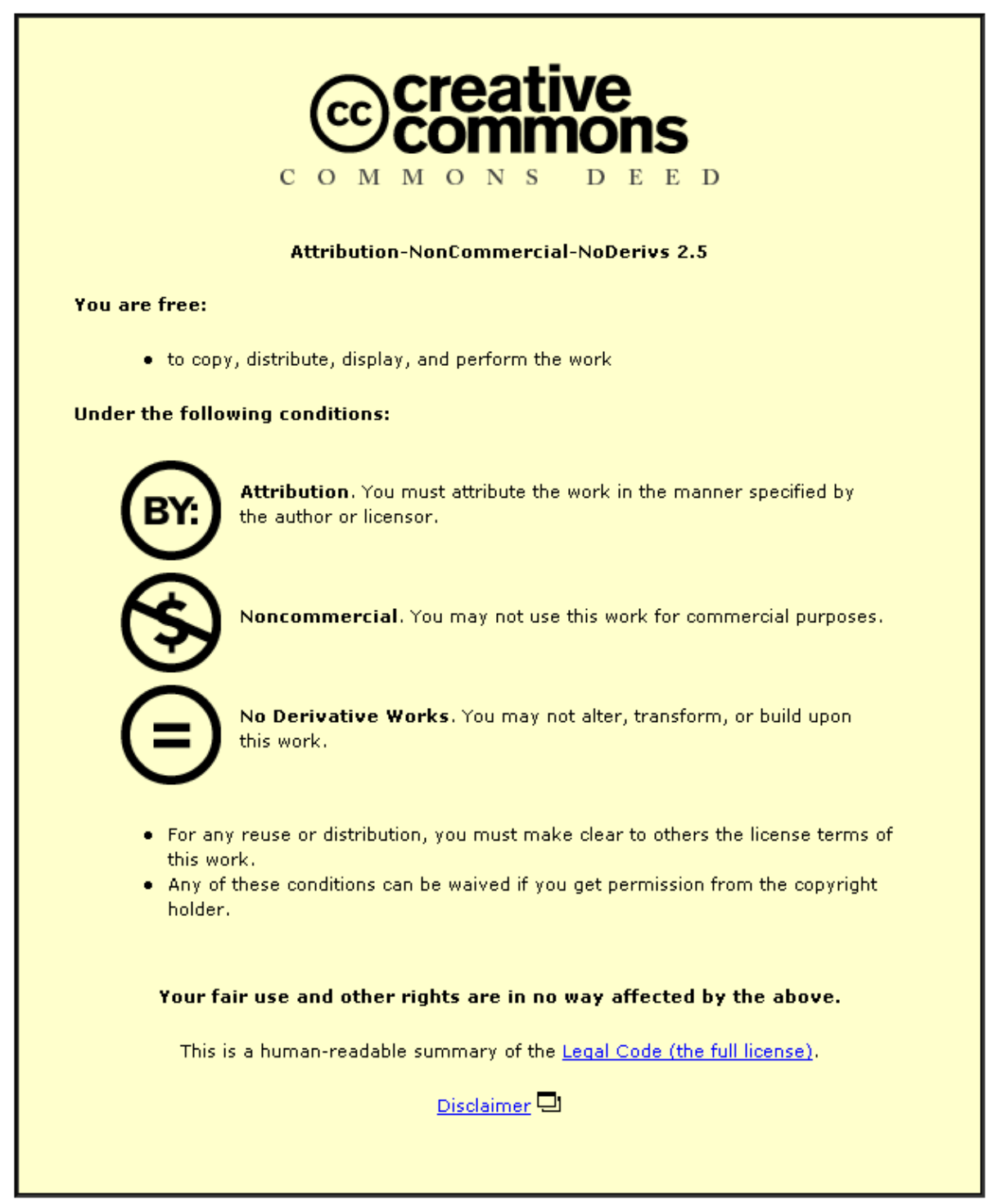

For the full text of this licence, please go to: http://creativecommons.org/licenses/by-nc-nd/2.5/ 


\title{
Normal incidence $X$-ray standing wave study of Fe on $\mathrm{Cu}(111)$
}

\author{
M T Butterfield and M D Crapper* \\ Department of Physics, Loughborough University, Loughborough, \\ LE11 3TU, UK.
}

The structure of annealed ultra-thin Fe films on $\mathrm{Cu}(111)$ has been investigated using normal incidence X-ray standing wave (NIXSW). Films of between 0.5 and 5.3 monolayer equivalence (MLE) have been deposited in situ and briefly annealed to $240^{\circ} \mathrm{C}$. NIXSW measurements of the $(111)$ and $(\overline{1} 11)$ reflections using $2 \mathrm{p}_{3 / 2}$ photoemission show that the film is pseudomorphic up to 2 MLE, with near complete occupation of the fcc hollow sites. For this regime, the value of the (111) coherent position is equivalent to a film spacing relative to the lattice of $2.00 \pm 0.03 \AA$. For the 5.3 MLE film, there is a drastic reduction in the $(\overline{111})$ coherent fraction and shift in the $(\overline{111})$ coherent position that is consistent with the transformation of the entire film to a Kurjumov-Sachs oriented bcc(110) structure.

Keywords: X-ray standing waves, growth, copper, iron, low index crystal surfaces, magnetic films, metal-metal interfaces, metal-metal magnetic thin film structure.

*Corresponding author 


\section{Introduction}

The possibility of using epitaxial growth to produce metastable phases of magnetic transition metals has been the focus of considerable attention within both the magnetism and surface science communities. In the case of Fe, which is bcc at room temperature, the use of closely matched substrates has enabled the production of (fcc) $\gamma$-Fe, which has a rich variety of magnetic properties, depending mainly upon the lattice spacing. One suitable substrate for the production of $\gamma-\mathrm{Fe}$ is fcc $\mathrm{Cu}$. The growth of $\gamma$-Fe has been studied on the (100) [1], (110)[2] and (111) [3-12] faces under a variety of conditions. Whilst it is known that growth on the (100) surface of $\mathrm{Cu}$ is well behaved, the growth on the closer packed (111) is more problematic.

The structural properties of ultra-thin films of Fe grown at room temperature on $\mathrm{Cu}(111)$ have been studied by a variety of methods, most notably low energy electron diffraction (LEED) $[3,4,5,6]$, X-ray photoelectron diffraction (XPD) [5], ultraviolet photoelectron diffraction (UPD)[7], scanning tunnelling microscopy (STM) $[8,9,10,11]$ and surface

EXAFS [12]. Most of these studies have also involved Auger electron spectroscopy (AES) to estimate coverage and determine growth mode. These reports have concentrated on the structure and morphology of the $\gamma$-Fe films, the thickness at which the transition to bcc Fe occurs, and the orientational relationship between the bcc overlayer and the fcc substrate. There is general agreement that at low coverage, at room temperature, 
the Fe grows in a pseudomorphic fcc structure. This has been demonstrated by LEED [3,4], XPD [5], UPD [7] and by Surface EXAFS [12] and has been implied by the appearance of triangular islands in STM images [10]. Early LEED/AES investigations reported layer-by-layer growth $[3,4]$, but more recent XPD [5] and STM [10] measurements have shown that the Fe grows in 3-D islands and that the initial islands are mostly of bilayer height. This has been attributed to high in-plane mobility but with a reduced probability of step-crossing for the Fe atoms. Structural determinations of the fcc Fe phase have given an interlayer spacings ranging from that of $\mathrm{Cu}(2.087 \AA)[6]$ to a contraction of $3-6 \%$ [5].

Upon increasing thickness, the room temperature Fe film undergoes a structural change to bcc, which has been confirmed by LEED [3], XPD [5], UPD [7] and surface EXAFS [12]. The bcc structure registers onto the fcc substrate in the Kurjumov-Sachs orientation with the bcc (110) normal to the surface. The bcc [111] troughs are aligned with [110] rows of fcc atoms. However, there is a deal of uncertainty about the critical thickness for this transformation with reports varying between 2 and 6 monolayers $(M L)$. It is now generally accepted that a transition occurs between 2 and $4 \mathrm{ML}$, with a true bcc structure being present by about 5-8 ML. Whether this transformation involves the entire film, or whether the bcc Fe registers onto fCc Fe is unclear. The XPD [5] and surface EXAFS [12] measurements indicated a transition region where neither fcc nor bcc was a good description, and that it was possible that domains of both were co- 
existing. The UPD [7] measurements, however, reported an abrupt transformation with the entire film converting to bcc. In contrast, STM images [10] show bright needle like areas that are considered to be needle like domains of bcc Fe sitting on fcc Fe.

Although in real device growth substrate temperature may be controlled to improve film properties, less is known about the temperature dependence of the growth of the system. It has been reported [8] that 3fold fcc islands grow at $423 \mathrm{~K}$ and $623 \mathrm{~K}$, but it may be that $\mathrm{Cu}$ segregates to the Fe surface at temperatures $[3,5]$ of $375 \mathrm{~K}$ or $473 \mathrm{~K}$. In the present work we have used the X-ray standing wave method to investigate films grown at room temperature, but then subjected to a flash anneal with the aim of improving order. The focus of the work is to determine the low coverage structure of such films, how the structural transformation to bcc will manifest in NIXSW, and whether the transformation to bcc involves the entire film.

The X-ray standing wave technique $[13,14]$ can be employed to determine the structure of adsorbates on metal and semiconductor surfaces as follows. The photon energy of a synchrotron radiation source is scanned through an X-ray Bragg reflection condition of the substrate. The incident and reflected waves interfere to form a standing wave, the nodes of which move in space as the photon energy is varied. The absorption profile of any atom through this photon energy range is characteristic of the position of the atom relative to the atomic planes of the substrate. The 
experiment may be conducted at any arbitrary incidence angle, but at normal incidence to the atomic planes the influence of mosaicity is minimised and typical metal crystals may be used [14]. When applied to the normal reflection of a surface, the method provides information about the position of an adsorbate relative to the extended bulk atomic planes. Using an off-surface-normal reflection enables a simple geometrical triangulation of the adsorption site.

When applied to several layers of metal-on-metal epitaxy, analysis of NIXSW is complicated since several different adsorption sites will be present. However, the parameters that are usually extracted from an NIXSW analysis, the coherent position and fraction, are informative about the interlayer separations, the degree of site order, and the incidence of stacking faults. We have applied NIXSW to the study of the growth of Fe films on $\mathrm{Cu}(111)$ for thicknesses of 0.5 to 5.3 monolayer equivalents (MLE). NIXSW clearly shows that in the sub 2 MLE regime, growth is pseudomorphic but by 5.3 MLE the film structure has undergone a drastic reorganisation.

\section{Method}

The experimental measurements reported in this work were made using station 6.3 [15] on the Synchrotron Radiation Source (SRS) at Daresbury Laboratory, UK. Station 6.3 is dedicated to NIXSW and SEXAFS measurements, and is equipped with a double-crystal $\mathrm{Ge}(111)$ monochromator capable of delivering X-rays in the energy region of the 
normal incidence $\mathrm{Cu}(111)$ Bragg reflection (2965 eV at room temperature). The station is equipped with a concentric hemispherical electron energy analyser for electron spectroscopy, which was used both for sample characterisation and to monitor the X-ray absorption of surface atoms during the NIXSW measurements. The base pressure remained below $1 \times 10^{-9}$ mbar throughout the experiment.

A Cu(111) crystal was prepared ex situ using the usual methods. In situ sample preparation consisted of $\mathrm{Ar}^{+}$ion bombardment and annealing to produce a clean and well-ordered surface as judged by X-ray photoelectron electron spectroscopy and low energy electron diffraction. Fe films were deposited in situ using a well-outgassed K-cell with the substrate at room temperature. Several thicknesses between 0.5 and 5.3 monolayer equivalents (MLE) were used, each being estimated by comparison of the intensities of the $\mathrm{Fe} 2 \mathrm{p}_{3 / 2}(\mathrm{BE}=706.8 \mathrm{eV})$ and $\mathrm{Cu} 2 \mathrm{p}_{3 / 2}$ $(\mathrm{BE}=932.5 \mathrm{eV})$ photoelectron peaks using a photon energy of $3000 \mathrm{eV}$. Following deposition, the sample was flash annealed to $\sim 240^{\circ} \mathrm{C}$ to improve film order. This temperature was selected as it has previously been shown to improve order, but without inducing the Fe to dissolve to form fcc inclusions in the bulk as can happen at higher temperatures [16].

NIXSW measurements were made of the Fe films using both the normal (111) and $70.2^{\circ}$ off normal $(\overline{1} 11)$ reflections. The X-ray energy was swept in steps of $0.2 \mathrm{eV}$ over a $20 \mathrm{eV}$ window centred on the $\mathrm{Cu}(111) \mathrm{Bragg}$ energy, and the absorption of both overlayer and substrate was monitored 
by determining the intensity of $X$-ray induced electron emissions; the $2 p_{3 / 2}$ photoemission in the case of $\mathrm{Cu}$, and both the $2 \mathrm{p}_{3 / 2}$ photoemission and LVV Auger emission for Fe $(K E=642 \mathrm{eV})$. For each photon energy, the electron emission intensities were determined by recording the difference in number of counts per second on the peak, and the background counts off the peak at slightly higher electron energy. For the thicker films, the monitoring of the Fe adsorption using the LVV Auger transition in addition to the $2 p_{3 / 2}$ transition enabled the data to be corrected for non-dipole effects [17].

\section{Results}

A selection of typical NIXSW profiles is displayed in Figure 1. These data show the Fe adsorption profile at the $\mathrm{Cu}(111)$ Bragg energy for a range of Fe coverages that have been subjected to a post-growth flash anneal.

Figure $1(a)$ shows the $(111)$ reflection and Figure $1(b)$ the $(\overline{1} 11)$ reflection. In this figure, the abscissa is the photon energy and the ordinate is the difference in counts on and off the $2 \mathrm{p}_{3 / 2}$ Fe photoemission peaks. Owing to a technical difficulty, the data for the 5.3 MLE film was collected using the Fe LVV alone. However, comparisons between data collected using both Fe LVV and $2 \mathrm{p}_{3 / 2}$ at the other thicknesses show no significant difference in the extracted parameters.

Quantitative analysis of the NIXSW profiles was carried out using the NIXSWFit [18] routine. This extracts two pieces of information from the 
profile, the coherent position and the coherent fraction. In the case of a single overlayer site, the coherent position is the z-spacing of the overlayer relative to the substrate continuation planes in units of the substrate d-spacing, and the coherent fraction reflects the degree of order. In systems where more than one type of site is occupied, as could be the case in ultra-thin films, the picture is not so simple and the values obtained for both parameters will be a function of the values for the individual sites as determined by equation 1 , where $F_{c}$ is the coherent fraction, $\mathrm{P}_{\mathrm{c}}$ the coherent position, $\mathrm{F}_{\mathrm{j}}$ the fractional occupation of the $\mathrm{j}^{\text {th }}$ site and $z_{j}$ is the $z$-coordinate of the $j^{\text {th }}$ site. The substrate layer spacing is $d$. It can be seen that the presence of different sites, perhaps corresponding to different layers of the structure or a phase change at a particular thickness, will reduce the coherent fraction. The degree of reduction in this parameter is sensitive to the $z$-coordinate of each individual site.

$$
F_{c} \exp \left(2 \pi i P_{c}\right)=\Sigma F_{j} \exp \left(2 \pi i z_{j} / d\right)
$$

The values of $\mathrm{F}_{\mathrm{c}}$ and $\mathrm{P}_{\mathrm{c}}$ extracted using NIXSWFit are shown in figure 2 . The abscissa in these plots is the number of Fe MLE and the ordinate is the value of the parameter. If $\mathrm{P}_{\mathrm{c}}$ is the coherent position expressed in the range 0 to 1 (where $P_{c}=1$ is equivalent to $z=d$ ) then there is no formal distinction between $P_{c}$ and $P_{c}+n$ where $n$ is an integer. This is because $P_{c}$ is simply the value of the position relative to the nearest expected position of the Cu scatterer planes extended out into space above the crystal surface. The values for $P_{c}(111)$ are shown as filled circles, those for 
$P_{c}(\overline{1} 11)$ as open circles, those for $F_{c}(111)$ as filled triangles, and those for $\mathrm{F}_{\mathrm{c}}(\overline{1} 11)$ as open triangles.

A similar NIXSWFit analysis was carried out for the emission from the $\mathrm{Cu}$ substrate. The values for coherent position $(1.00 \pm 0.01)$ and fraction $(0.90 \pm 0.02)$ were found to be in the range expected for a $\mathrm{Cu}(111)$ surface [19]. The reduction of this coherent fraction from 1.00 is larger than that that could be expected from a typical r.m.s. thermal deviation of $0.07 \breve{A}$ for $\mathrm{Cu}$, so its source is most likely static disorder.

\section{Discussion}

The data for the annealed films should be considered in two regimes. The first is in the thickness range 0-2 MLE, where all reports agree that the room temperature grown film is pseudomorphic fcc. The second regime is above 2.0 MLE as it is known that the transition to bcc occurs in the $2-8$ MLE range for the room temperature structures.

Taking the low thickness regime, the first point to note in Figure 2 relates to the values for $P_{c}(111)$, the coherent position in the normal reflection. In each case, this is less than 1 indicating that the positions of the Fe atoms are slightly contracted over the expected $\mathrm{Cu}(111)$ bulk continuation. The spread of values for $P_{c}(111)$ is 0.95 to 0.97 (each \pm 0.01 ) but there is no discernable trend with film thickness. This indicates a Fe position of $2.00 \pm 0.03 \AA$ compared with a $\mathrm{Cu}(111) \mathrm{d}$-spacing of $2.087 \AA$. This value is 
in agreement with the UPD work [7] where an Fe-Cu distance of $1.99 \pm 0.03 \AA$ was reported for 0.5 MLE grown at room temperature indicating no change in position on annealing. Note, however, that UPD and NIXSW measure slightly different things; the positions quoted in UPD are relative to the emitting atom (Fe) and those in NIXSW relate to the ideal bulk lattice continuation. It is known that the surface layer of clean $\mathrm{Cu}(111)$ relaxes, which if it occurs in Fe covered $\mathrm{Cu}(111)$ will be a contributing factor to the apparent Fe-Cu distance measured by NIXSW. If the Fe interlayer spacing in ultra-thin films were less than that of the $\mathrm{Cu}(111)$ direction, then a continuing trend of decreasing $\mathrm{P}_{\mathrm{c}}(111)$ would be expected with a gradient of half of the value of the difference in Fe-Fe and Cu-Cu spacing. In our measurements of up to only $2 \mathrm{MLE}$, such a slope is not detectable. If it existed, it would be at less than the experimental error.

In Figure 2, note that the values of $F_{c}(111)$ decrease slightly with film thickness. There are three possible causes of such a decrease; the first is thermal disorder, the second is static disorder, and the third is multiple site occupation perpendicular to the surface. The third cause may be discounted as a fit of the values would require a $5 \%$ occupation of a site with a $25 \%$ increased $z$-spacing. The decrease on the $F_{c}(111)$ is much less than that recently reported for $\mathrm{Mn}$ on $\mathrm{Cu}(111)$ [20], where there is very strong evidence for multiple site occupation. The magnitude of the may be explained for some of the data points by thermal disorder, by allowing for a doubling of the r.m.s. displacement found in bulk Fe to take account for 
the reduced dimensionality and adding in quadrature, the r.m.s. displacement of the bulk $\mathrm{Cu}$ atoms that set up the wavefield. However, it is likely that static disorder is at least partly involved. Evidence for static disorder in the un-annealed films is given by measurements of $F_{c}(111)$ for 1.7 ML before and after annealing, where it was found that the coherent fraction increased from 0.66 to 0.83 , whilst the coherent position remained unchanged at 0.95 . The presence of disorder in un-annealed films ties in with SEXAFS measurements of the system, where the number of nearest neighbours was found to decrease with increasing MLE.

Insight into the site occupation of the Fe atoms in the low coverage case can be obtained from the values for $F_{c}(\overline{1} 11)$ and $P_{c}(\overline{1} 11)$. NIXSW measurements made using the near grazing $(\overline{1} 11)$ reflection are very sensitive to the adsorption site parallel to the surface with the three high symmetry sites, the fcc hollow, the hcp hollow, and the atop site, having very different signatures. The values for the coherent position for this reflection, $\mathrm{P}_{\mathrm{c}}(\overline{1} 11)$, stay more-or-less constant throughout the low coverage range, indicating that the average site position relative to the $(\overline{1} 11)$ reflection is constant and close to one. Of course, this parameter would be complicated by multiple site occupation and the existence of domains. However, when used in combination with the coherent fraction, $\mathrm{F}_{\mathrm{c}}(\overline{111})$, this parameter can be used to determine the adsorption site occupations in a quantitative way. In Figure 2, it can be seen that in the range up to 2 MLE there is very little change in $F_{c}(\overline{111})$, indicating single 
site occupation. In combination with $\mathrm{P}_{\mathrm{c}}(\overline{11} 11)$, this indicates that there is little deviation from the expected fcc substrate continuation.

The relationship between $\mathrm{P}_{c}(\overline{1} 11)$ and $\mathrm{F}_{\mathrm{c}}(\overline{111})$ can be better visualised by using the Argand plane construction. In this scheme, which is depicted in Figure 3, the sites are represented by vectors with angle relative to the real axis of $\theta=2 \pi P_{c}(\overline{1} 11)$ and of length $F_{c}(\overline{1} 11)$. In Figure 3 for increased clarity we have used the ratio of $F_{c}(\overline{111})$ for Fe to that of $\mathrm{Cu}$ in order to remove the effect of intrinsic substrate disorder, but this modification does not alter the conclusions that may be drawn. The three high symmetry sites are represented by vectors at different angles. $100 \%$ occupation of the fcc hollow would result in a vector near to the positive real axis. The slight deviation from the axis reflects the expected zspacing contraction revealed by the $\mathrm{P}_{\mathrm{c}}(111)$ value. The hcp hollow, however, has a very different $(\overline{1} 11)$ z-spacing resulting in a vector in the third quadrant. The vector corresponding to the atop site is in the second quadrant. Inspection of the data points for 0.5-2 MLE indicates that the film is dominated by the occupation of the fcc hollow, but with an increase in thermal and static disorder over the substrate revealed by the slight reduction in $\mathrm{F}_{\mathrm{c}}(\overline{1} 11)$.

At this point, it is instructive to consider the effect of the occupation of multiple sites. In the Argand scheme, this would be represented by the addition of two vectors, the angle of each being determined by the 
respective $z_{111}^{-}$-spacing relative to the $\mathrm{Cu} \mathrm{d}_{111}^{-}$-spacing and their length being the product of the site occupation and the substrate $F_{c}(\overline{111})$. For example, 50\% occupation of the fcc and hcp hollows would result in a vector midway between the two, but of length reduced to one half. Thus any reduction in $F_{c}(\overline{1} 11)$ could reflect disorder, or multiple site occupation, or both. The reduction in $F_{c}(\overline{1} 11)$ seen in the low coverage data is most probably due to disorder, as to explain it by multiple site occupation would require about a $5 \%$ occupation of the atop site. The direct occupation of the atop site in metal-on-metal epitaxy is unlikely, but it may be mimicked in the global perspective of the NIXSW technique by the incorporation of two stacking faults as could occur if the film were twinned. The effect of a twin would be to give equal occupation of the hcp hollow and the atop site, an effect illustrated in Figure 4 and Table 1. Figure 4 shows schematically the abcabc stacking that is characteristic of the fcc lattice in the (111) direction. Should the overlayer grow in an fcc registered twin, however, the sequence would be acb instead of abc, which is also shown in Figure 4. The occupation of the c position in the second layer would be an hcp hollow both locally and with respect to the $\mathrm{Cu}$ lattice continuation. The occupation of site b in the third layer, however, is locally an fcc hollow, but relative to the $\mathrm{Cu}$ lattice continuation is an atop. Table 1 lists the NIXSW site designation of each position versus layer number. To explain the reduction in $F_{c}(\overline{1} 11)$ seen here would require a twinning fraction of $\sim 5 \%$ in the submonolayer range, rising to $\sim 15 \%$ in the $1-2$ MLE range. This however, would not explain why there 
tends to be a slight favouring of the atop site over the hcp hollow.

The second regime to consider corresponds to films greater than 2 MLE thickness. It is in this range that the conversion of the film to bcc is reported for the room temperature films. For the annealed films, the two data points in this range do show some differences to the thinner films. For the 2.6 MLE equivalent film shows a slight reduction in $P_{c}(\overline{1} 11)$ that may indicate the onset of a structural change. The 5.3 MLE film, however, differs drastically from the other films. It displays a reduction in $\mathrm{P}_{c}(\overline{111})$ to $0.84 \pm 0.02$ and a massive reduction in $F_{c}(\overline{1} 11)$ to $0.13 \pm 0.04$. The reduction in $F_{c}(\overline{111})$ indicates an almost total loss in site coherence with the substrate that may be understood as follows.

A fourth high-symmetry adsorption site on the fcc(111) surface may be described as a two-fold bridge site. The presence of such a two-fold site on a three-fold surface would result in three domains that may be mapped onto each other by successive rotations of $120^{\circ}$. The effective $P_{c}(\overline{111})$ of the combination of the three domains of bridge sites is $z(111) / 3+d(111) / 2$, where $z(111)$ is the adsorbate (111) spacing and $d(111)$ is that of the substrate. The combined effective $F_{c}(\overline{111})$ is $F_{c}(111) / 3$ as three sites are involved. The vector for a direct occupation of the bridge site lies midway between those representing the two hollows in the fourth quadrant of the Argand diagram, shown in Figure 3. 
In bcc(110) stacking, each successive layer does stack into a bridge site. An undistorted KS registered bcc(110) structure would produce six domains in mirror image pairs with only a slight difference in alignment, each pair separated by $120^{\circ}$. However, owing to the small angular difference between the pairs and the strain that is undoubtedly present, it may be assumed that the bcc lattice is distorted to fit the fcc sites for the purposes of the interpretation of the NIXSW data without introducing any major error. Figure 4 shows a schematic of bcc(110) stacked on fcc(111) in the KS orientation projected onto the fcc [ $\overline{2} 11]$ and the bcc $[\overline{11} 12]$ azimuthal directions. In this scheme, the first, third and fifth layers locate into fcc a-type positions giving "fcc", "hcp" and "atop", and all even layers locate into a local bridge site. This is illustrated in Figure 4, but note that the atoms are not in b sites, but are displaced into the page behind the plane. These local bridge sites have different $z(111)$ values as they are in different layers. This causes the expected $\mathrm{P}_{c}(111)$ to precess around the Argand with layer number in the same manner that the "a" site may be regarded as fcc, hcp or atop depending upon which layer it is in. In Figure 3, we have shown the vector representations of these sites. In each case the coherent fraction is 0.33 , but the coherent position varies by $\pm 2 \pi / 3$. The first bridge site (on an fcc registered layer) has $P_{c}(\overline{111})$ midway between fcc and hcp sites. The second bridge site on a non-fcc registered layer, two layers further up, has $\mathrm{P}_{\mathrm{c}}(\overline{1} 11)$ midway between hcp and atop. The final bridge site, two layers further up again, has $P_{c}(\overline{111})$ midway between atop and fcc. 
A simplistic model of $\operatorname{bcc}(110)$ in the KS orientation on $\mathrm{fcc}(111)$ would involve $17 \%$ of the atoms in each of these bridge sites, $17 \%$ in fcc, $17 \%$ in hcp, and $17 \%$ in atop. Thus, the expected coherent parameters for this structure are $P_{c}(\overline{11} 1)$ undefined and $F_{c}(\overline{11} 1)=0$. However, this assumes equal occupation of each layer. STM images of the room temperature system show that the occupation decreases with increasing layer number. If a similar layer filling pattern occurs in the annealed films, then in an fcc registered KS bcc Fe structure, the fcc and first bridge site (No. 1) will be over-represented and the atop and last bridge site (No. 3) will be underrepresented. So, instead of a coherent fraction of 0 and undefined coherent position, the bcc overlayer would be expected to give values with a small coherent fraction and the coherent position in the fourth quadrant. This is precisely what we see for the 5.3 MLE film. The coherent parameters of the 5.3 MLE film are fitted very well by a crude model involving $27 \%$ occupation of fcc like sites followed by a $4 \%$ decrease to each successive site in the order bridge 1, hcp, bridge 2, atop, bridge 3. In reality, the strain in the epitaxial lattice will disturb the structure increasingly with layer number, so the truth will be more complex.

Our data are consistent with a complete conversion of the annealed film to bcc by 5.3 MLE. However, there remains the question as to whether there really is a complete conversion of the film, or whether the bcc structure sits upon an interface structure of 2 MLE or more of fcc Fe. In fact, the conversion can be regarded to be complete, as is indicated by the parameter that shows least scatter, the coherent position. For the 5.3 
MLE film this is $0.84 \pm 0.02$, but if the structure started with two fcc like layers followed by bcc stacking it would be expected to be 0.99 , though the coherent fraction would indeed be similar at 0.14 . Allowing for unequal filling of all the sites then this could be reduced to 0.91 , still well outside the confidence limits. In addition, this latter case would increase the coherent fraction to 0.25 and is considered to be highly unlikely given the mismatch of the film and substrate, which is known to cause islanding in the room temperature grown film. Figure $1(b)$ shows a grey line imposed on the 5.3 MLE data that shows the profile that would be expected if the bcc Fe were sited on two layers of fcc Fe.

\section{Conclusion}

NIXSW has been employed to investigate the structure of post-growth flash annealed ultra-thin films of Fe grown on $\mathrm{Cu}(111)$. In the regime $0-2$ MLE of Fe, it was found that the (111) spacing of the Fe contracts with respect to the $\mathrm{Cu}(111)$ spacing, to an average value of $2.00 \pm 0.03 \AA$ relative to the substrate continuation. In this low coverage regime, the NIXSW shows that the fcc site is predominantly occupied, consistent with pseudomorphic fcc growth. At a higher coverage of $5.3 \mathrm{MLE}$, there is a drastic change in the coherent parameters extracted by NIXSW that is consistent with a relaxation of the entire film to a bcc like structure somewhere above 2 MLE. It has been shown that NIXSW is a useful tool in the study of metal-on-metal epitaxy, enabling quantitative structural information to be determined in the pseudomorphic and clearly 
fingerprinting a change in structure at higher coverages.

\section{Acknowledgements}

This work was supported by the EPSRC via the provision of access to the SRS and a studentship for MTB. At the SRS, George Miller provided technical support and Vinod Dhanak scientific support. 


\section{References}

[1] See for example A. Biedermann, R. Tscheliessnig, M Schmid and P Varga, Physical Review Letters 87 (2001) 086103.

[2] See for example S Tacchi, F Bruno and G. Carlotti, Surface Science 507 (2002) 324.

[3] D. Tian, F. Jona and P. M. Marcus, Physical Review B 45 (1992) 11216.

[4] Y. Darici, J. Marcano, H. Min and P. A. Montano, Surface Science 195 (1988) 566.

[5] M. T. Kief and W. F. Egelhoff Jr., Physical Review B 47 (1993) 10785.

[6] G. Gubbiotti, L. Albini, g. Carlotti, S. Loreti, C. Minarini, and M. De Crescenzi, Surface Science 433-435 (1999) 680.

[7] A. Theobald, O. Schaff, C. J. Hirschmugl, V. Fernandez, K.-M. Schindler, M. Polcik and A. M. Bradshaw, Physical Review B 59 (1999) 2313.

[8] A. Brodde, K. Drepps, J. Binder, Ch. Lunau and H. Neddermeyer, Physical Review B 47 (1993) 6609.

[9] J. Shen, M. Klaua, P. Ohresser, H. Jenniches, J. Barthel, Ch. V. Mohan, and J. Kirschner, Physical Review B 56 (1997) 11134.

[10] P. Ohresser, J. Shen, J. Barthel, M. Zheng, Ch. V. Mohan, M. Klaua and J. Kirschner, Physical Review B 59 (1999) 3696.

[11] M. C. G. Passeggi Jr., J. E. Prieto, R. Miranda, and J. M. Gallego, Surface Science 462 (2000) 45.

[12] M. T. Butterfield and M. D. Crapper, Surface Science 454-456 (2000) 719.

[13] J. Zegenhagen, Surface Science Reports 18 (1993) 199.

[14] D. P. Woodruff, Progress in Surface Science 57 (1998) 1.

[15] A. A. McDowell, D. Norman, J. B. West, J. C. Campuzano, R. G. Jones, Nuclear Instrumentation and Methods A246 (1986) 131.

[16] M. T. Butterfield and M. D. Crapper, to be published.

[17] J. J. Lee, C. J. Fisher, D. P. Woodruff, M. G. Roper, R. G. Jones and B. C. C. Cowie, Surface Science 494 (2001) 166.

[18] NIXSW99\# 1, R. G. Jones, Dept. of Chemistry, University of Nottingham, Nottingham, UK.

[19] R. G. Jones, A. S. Y. Chan, M. G. Roper, M. P. Skegg, I. G. Shuttleworth, C. J. Fisher, G. J. Jackson, J. J. Lee, D. P. Woodruff, N. K. Singh and B. C. C. Cowie, Journal of Physics: Condensed Matter 14 (2002) 4059.

[20] M T Butterfield, M D Crapper and V R Dhanak, Physical Review B accepted. 


\section{Figure Captions}

Figure 1: Typical NIXSW profiles of the flash annealed Fe films on $\mathrm{Cu}(111)$ obtained from the (a) (111) reflection and (b) (111) reflection. The data were collected using the $\mathrm{Fe} 2 \mathrm{p}_{3 / 2}$ photoemission signal or (5.3 MLE) the $\mathrm{Fe}$ LVV Auger. The solid line shows the NIXSWFit fit to the data and the grey line for the 5.3 MLE data shows the curve expected if two monolayers of fcc Fe underlie a bcc structure.

Figure 2: The values for the coherent position (circles) and the coherent fraction (triangles) extracted for the (111) reflection (filled) and the ( $\overline{111})$ reflection (open). The values for the coherent fraction are expressed as fractions of the $\mathrm{Cu}(111)$ layer spacing.

Figure 3: A polar plot of the data $(\odot)$ for the $(\overline{111})$ reflection with $\theta=2 \pi \mathrm{P}_{\mathrm{c}}$ and $r=F_{c}(F e) / F_{c}(\mathrm{Cu})$. The expected values for the fcc hollow ( $\left.\mathbf{\Delta}\right)$, hcp hollow $(\boldsymbol{\nabla})$, atop $(\bullet)$ and bridge $(\boldsymbol{\nabla})$ sites are also shown.

Figure 4: Schematic of the stacking pattern cross-sectioned through the (211) azimuthal direction. This shows the a-b-c stacking pattern expected of an fcc continuation and the approximate deviation from this caused by a change to $b c c(110)$ stacking (one domain only). 


\section{Tables}

Table I. NIXSW site designation relative to an fcc continuation for the three fcc stacking positions shown in Figure 4.

\begin{tabular}{|c|c|c|c|c||}
\hline Layer & a & b & c & $\begin{array}{c}\text { Substrate } \\
\text { Continuation }\end{array}$ \\
\hline 1 & fcc & hcp & atop & A \\
\hline 2 & atop & fcc & hcp & B \\
\hline 3 & hcp & atop & fcc & C \\
\hline 4 & fcc & hcp & atop & A \\
\hline 5 & atop & fcc & hcp & B \\
\hline
\end{tabular}


Figure 1

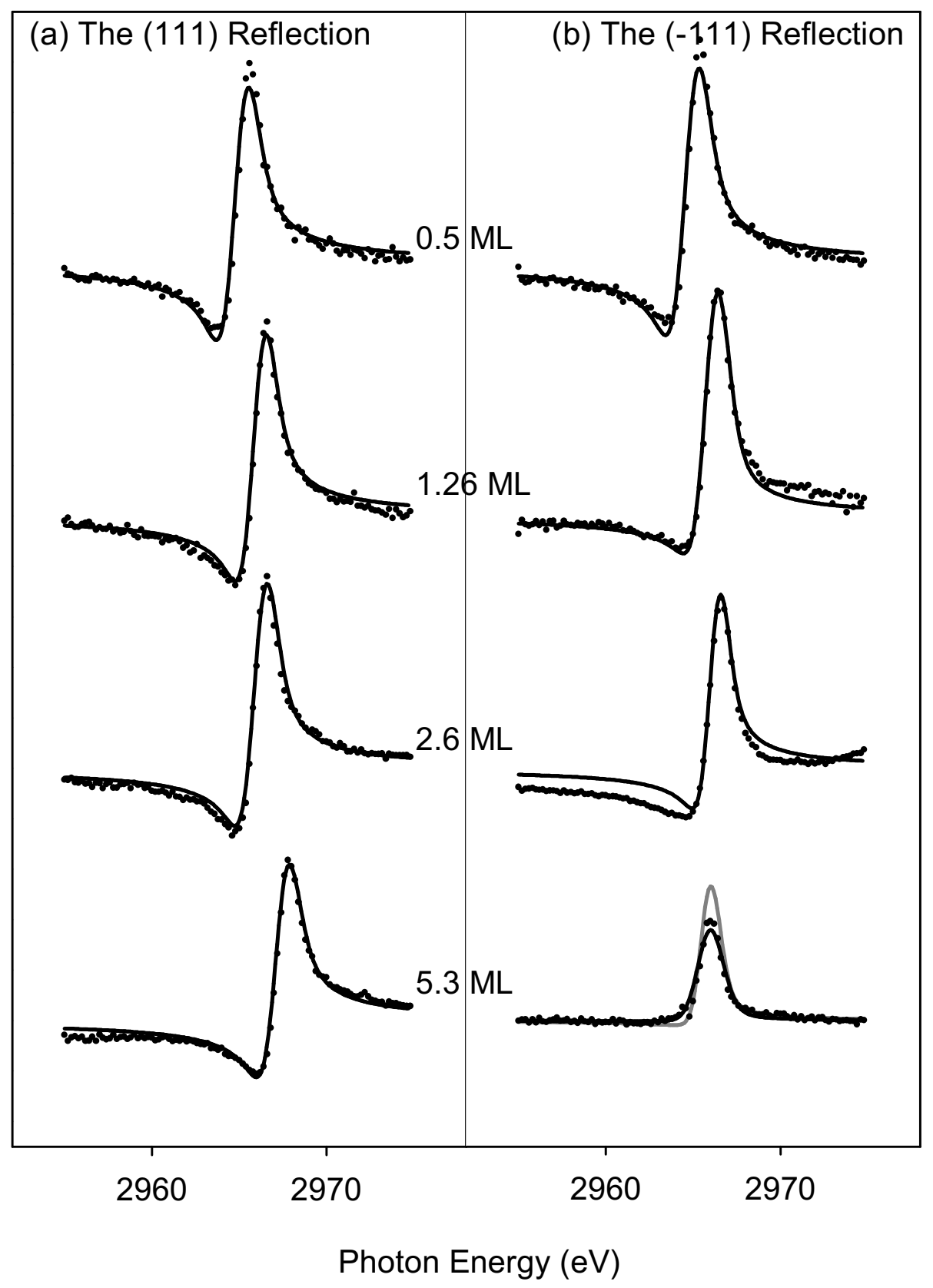


Figure 2

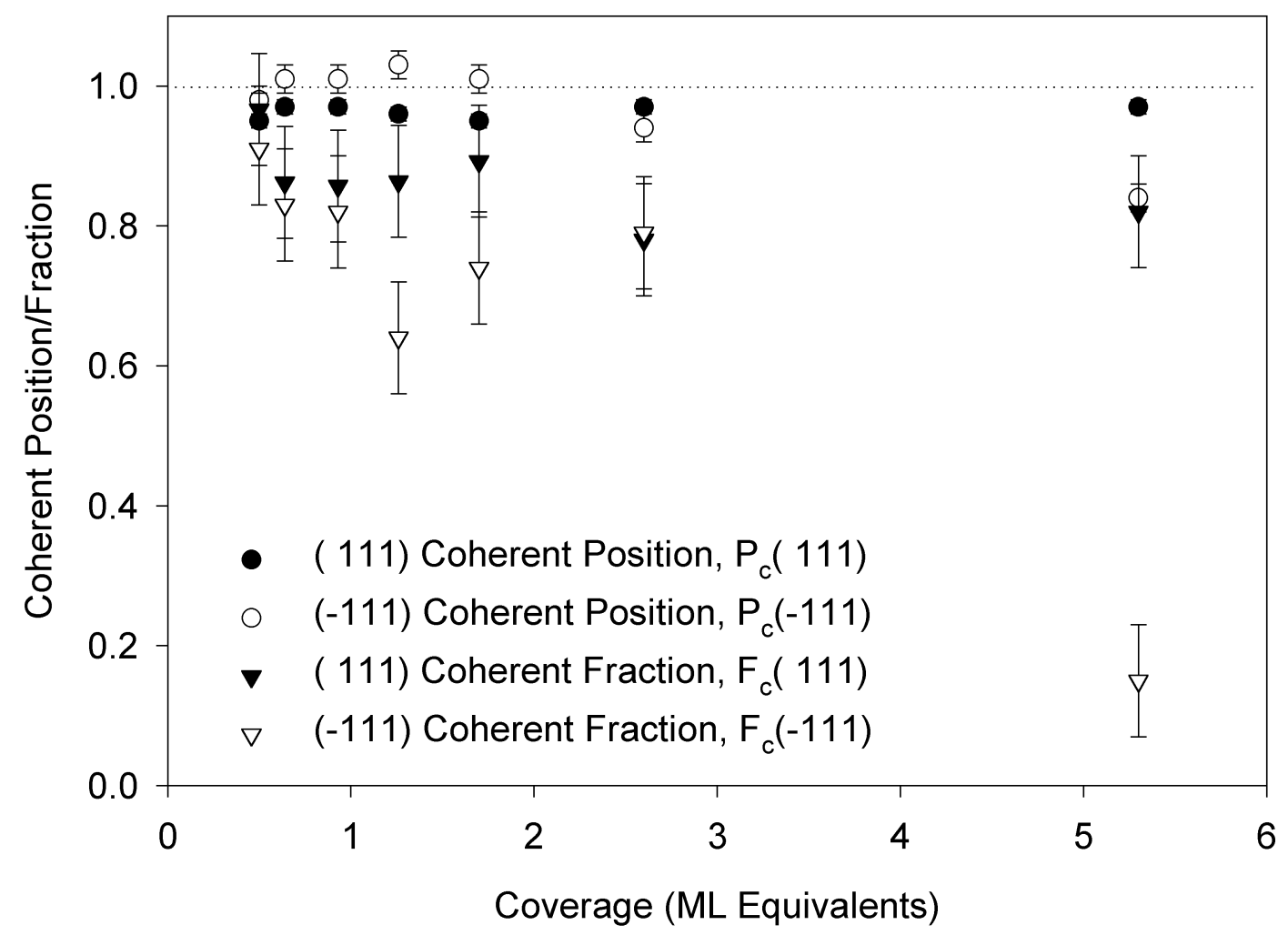


Figure 3

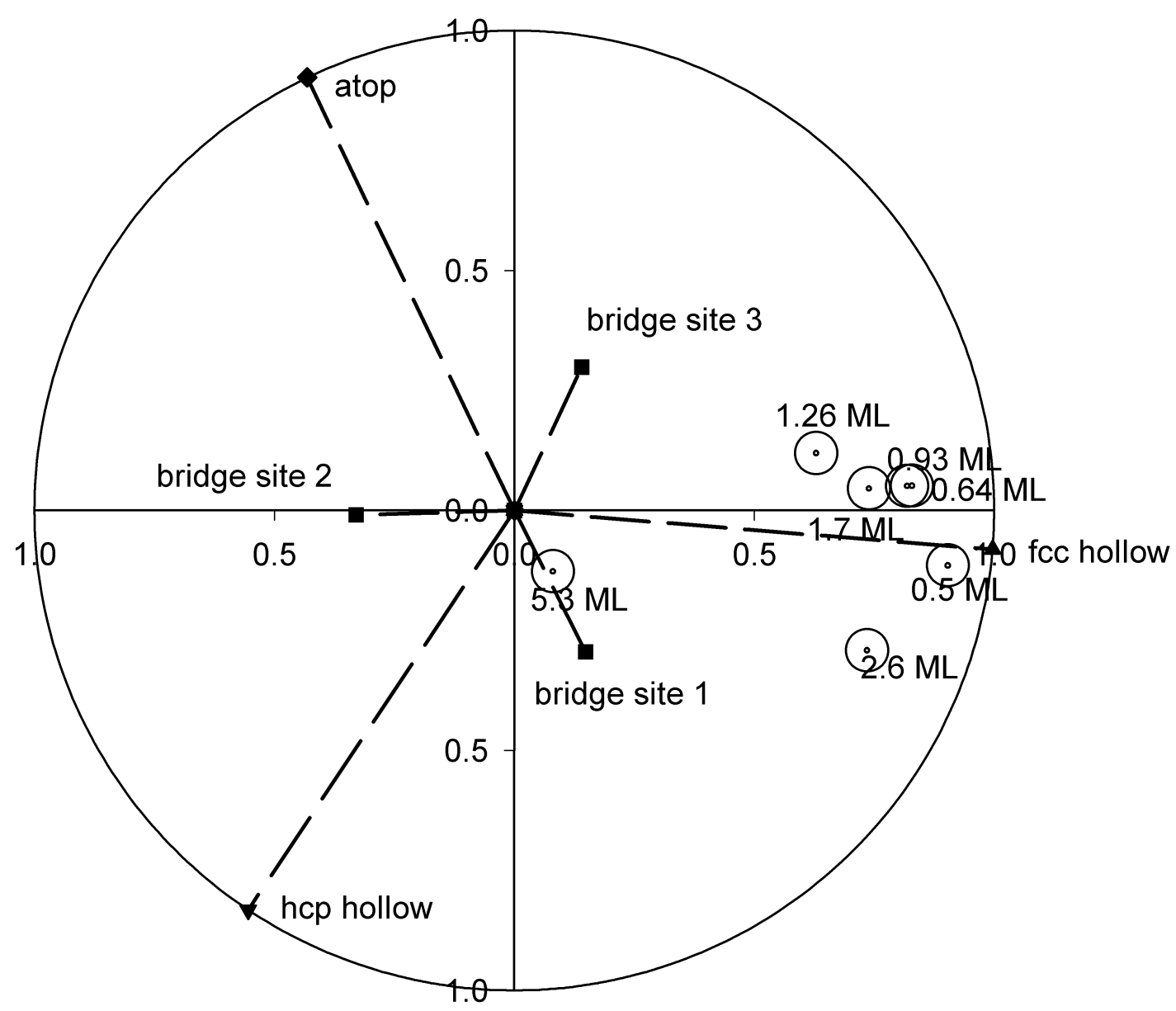


Figure 4

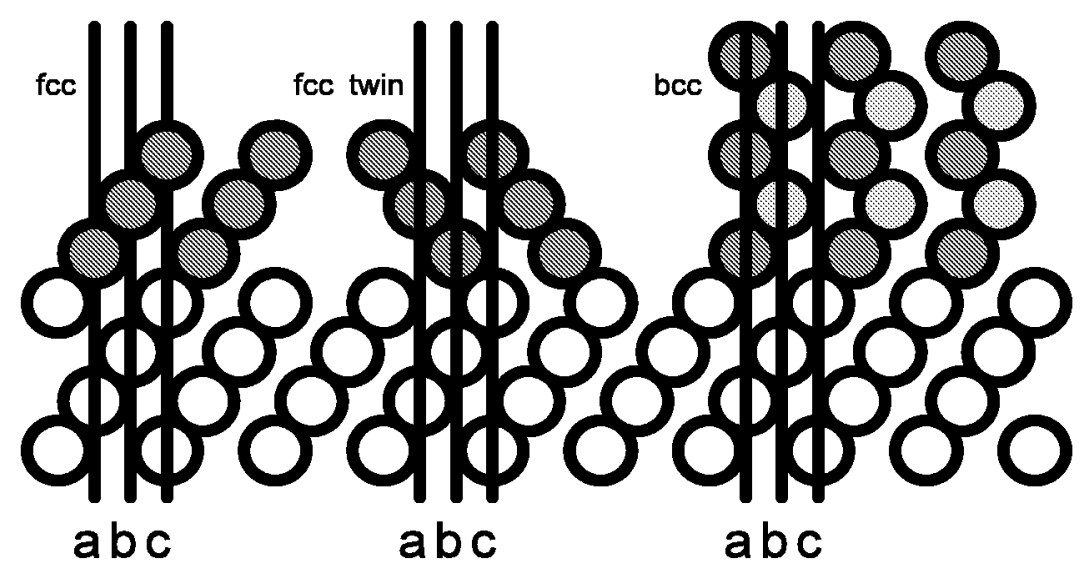

